\title{
Rare cause of rapidly progressive dementia - Creutzfeldt-Jakob disease (CJD) with negative 14-3-3 protein - Case Report
}

\author{
Marcin Bąk', Anna Jamroz-Wiśniewska², Małgorzata Nowakowska', Marcin Ciesielka', \\ Magdalena Sałek', Aleksandra Rawska', Konrad Rejdak ${ }^{2}$ \\ 1 Student Research Group of Neurology, Medical University, Lublin, Poland \\ ${ }^{2}$ Chair and Department of Neurology, Medical University, Lublin, Poland
}

Bąk M, Jamroz-Wiśniewska A, Nowakowska M, Ciesielka M, Sałek M, Rawska A, Rejdak K. Rare cause of rapidly progressive dementia Creutzfeldt-Jakob disease (CJD) with negative 14-3-3 protein. J Pre-Clin Clin Res. 2019; 13(3): 134-136. doi: 10.26444/jpccr/110431

\begin{abstract}
Sporadic Creutzfeldt-Jakob disease (sCJD) is the most common form of prion diseases. Although almost 100 years have passed since this disease was first described, specific treatment still does not exist. The case is presented of a 57-year-old woman, initially diagnosed with ischemic stroke. On the day of admission to hospital she presented non-specific symptoms (speech disorders, visual disturbances). During hospitalization, the patient presented rapidly progressive dementia. Later, new symptoms, such as blindness, akinetic mutism, rigidity and myoclonus appeared. With the progression of the disease, the patient developed characteristic MRI and EEG changes for SCJD. After excluding other potential causes, the diagnosis of probable SCJD was made. However, the result of 14-3-3 protein in the cerebrospinal fluid (CSF), characteristic for CJD, was negative. This case report highlights the importance and difficulties in differential diagnosis of rapidly deteriorating cognitive function disorder.
\end{abstract}

\section{Key words}

prion diseases, CJD (Creutzfeldt-Jakob Disease), 14-3-3 proteins, neurologic deficits

\section{INTRODUCTION}

Creutzfeldt-Jakob disease (CJD) is a neurodegenerative disorder, which belongs to the group of prion diseases. It was first described independently by Alfons Maria Jakob and Hans Gerhard Creutzfeldt in 1920 [1]. Prions are considered to be infectious particles that present as an abnormal, proteaseresistant isoform of a cellular protein - the prion protein or PrPC. The aggregates of misfolded protein conformers are depositing in the CNS of affected individuals [2]. Four human prion diseases are currently recognized: CreutzfeldtJakob disease (CJD), Kuru, Gerstmann-Sträussler-Scheinker syndrome (GSS), and fatal familial insomnia (FFI) [3]. Actually, four forms of CJD can be distinguished: sporadic (sCJD), familial (fCJD), iatrogenic (iCJD) and variant (vCJD). Sporadic CJD accounts for more than $90 \%$ of prion diseases; although it is still extremely rare, approximately one case occurs per 1,000,000 population per year, with a worldwide distribution [4].

\section{CASE REPORT}

In March 2018, a 57-year-old woman was admitted to the Neurology Department complaining of blurred vision, colour vision deficiency, speech disorders and walking abnormalities. Neurological examination revealed positive Romberg's test, dysarthria and psychomotor retardation, the patient was in logical contact with normal gait. The patient had been treated because of arterial hypertension

Address for correspondence: Anna Jamroz-Wiśniewska, Chair and Department of Neurology, Medical University of Lublin, Poland

E-mail: anna.jamroz@umlub.pl

Received: 04.05.2019; accepted: 02.07.2019; first published: 16.07.2019 and asthma. She underwent two lacunarischemic strokes, the first stroke (in the right occipital lobe) occurred in 2017, the second in February 2018 (in the right parietal lobe). Input CT didn't reveal any focal changes or intracranial bleeding. Basic laboratory tests were within normal limits.

During hospitalization, the condition of the patient deteriorated rapidly. Described symptoms intensified and new symptoms appeared - blindness and dementia with severe allo- and auto-psychic disorientation. Patient became also more sleepy during the day and refused to eat. Finally, akinetic mutism developed, the patient became bed-ridden, rigidity and generalized myoclonus appeared.

A number of tests were carried out to discover the cause of dementia: TSH level, ceruloplasmin level, vitamin B12 level $(=477 \mathrm{pg} / \mathrm{ml})$, level of anti-thyroid peroxidase and anti-thyreoglobulin antibodies - all came back negative. Diagnostic process showed elevated ANA, but SLE-specific markers were negative.

All serological tests for syphilis, AIDS, hepatitis B and $\mathrm{C}$, borreliosis, toxoplasmosis, CMV infection, anty-NMDA encephalitis, were negative. In addition, the patient was negative for tumour markers, including: CA 125, CA 15-3, CA 19-9. Onconeural antibodies were also negative.

Lumbar puncture was performed shortly after admission. There were no changes suggesting neuroinfection. Protein 14-3-3 in the CSF was not detected, which is why other causes of progressive neurological deterioration were considered.

In the first MRI, there were no specific lesions in the CNS, they were visualized only in the third MRI. The diffusionweighted imaging (DWI) MRI revealed a hyperintense signal in the posterior part of thalamus and lack of increased intracranial pressure symptoms. FLAIR and T2-weighted sequences showed increased signal intensity in the bilateral caudate nucleus, putamen and grey matter of parietal, 


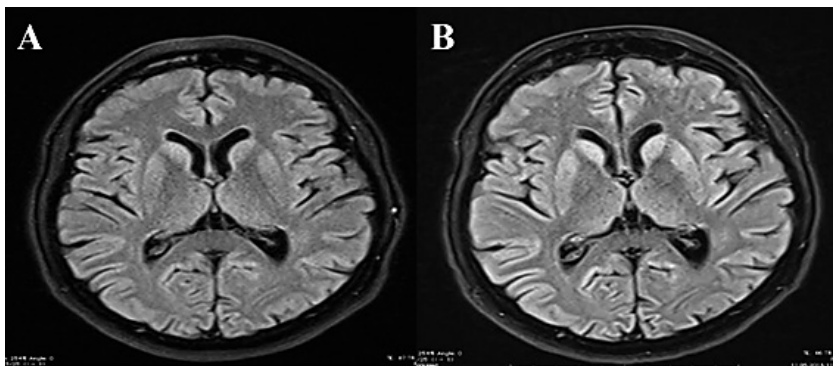

Figure 1.

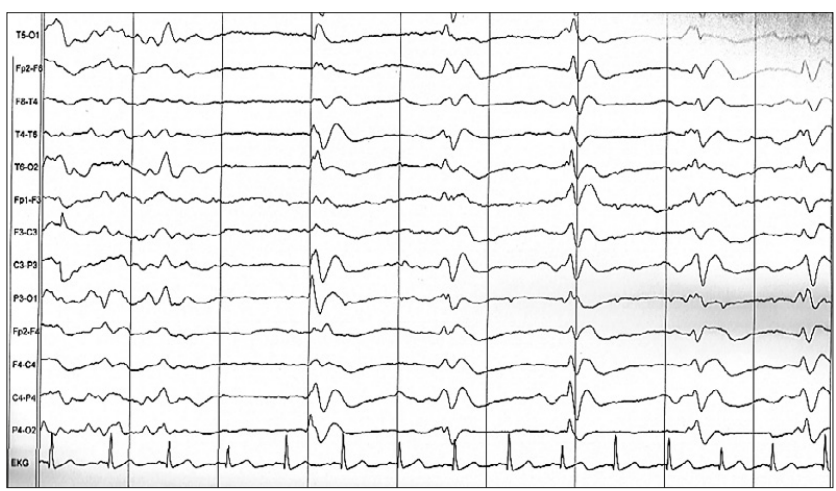

Figure 2.

tempora and, occipital lobes (Fig. 1). These findings appeared to fulfill the radiological diagnostic criteria of CJD.

Repeated electroencephalogram (EEG) revealed periodic sharp wave complexes (triphasic sharp theta wave) and sharpand-slow-wave complexes (Fig. 2). These abnormalities also suggested CJD.

All these findings allowed recognition of CreutzfeldtJakob disease. Despite intense treatment, patient died due to infection and respiratory failure. After the patient's death, no autopsy was not carried out due to fulfilled diagnostic criteria and the lack of family consent.

\section{DISCUSSION}

There is considerable variability in the clinical presentation of CJD which can make the initial diagnosis difficult. The disease usually occurs in middle- and older-aged patients, and typically affects individuals in their seventies, slightly more often in women [5]. Mean survival time ranges from 5-8 months [6-7]. In the presented case, the patient was 57 -years-old and survived only 3 months after admission to the Neurology Department.

The doctor must maintain a broad differential diagnosis at the onset. This includes, but is not limited to, neurodegenerative, vascular, autoimmune, infectious, toxic/metabolic, metastatic/neoplasmatic, and iatrogenic etiologies. In the presented case of SCJD, the initial diagnostic workup ruled out an array of known causes, including CJD.

According to diagnostic criteria (Tab. 1), there are three levels of diagnostic certainty of sCJD: definite, probable, and possible [8]. Definite diagnosis can be only made by neuropathological or immunocytochemical confirmation.

Common findings of sCJD in neurological examination include, but are not limited to, rapidly progressive cognitive
Table 1.

\begin{tabular}{ll}
\hline II & Rapidly progressive cognitive impairment \\
& $\begin{array}{l}\text { A. Myoclonus } \\
\text { B. Visual or cerebellar problems } \\
\text { C. Pyramidal or extrapyramidal features } \\
\text { D. Akinetic mutism }\end{array}$ \\
\hline III & Typical EEG (generalised periodic complexes) \\
\hline IV & High signal in caudate/putamen on MRI brain scan or least 2 \\
& cortical regions (temporal, parietal, occipital), either on DWI or \\
& FLAIR \\
\hline Possible CJD & I +2 of II + duration < 2 years \\
\hline Probable CJD & 1.2 .1 I + 2 of II and III \\
& or: \\
& 1.2 .2 I + 2 of II and IV \\
or: & 1.2 .3 I + 2 of II and positive 14-3-3 \\
or: & 1.2 .4 Progressive neurological syndrome and positive RT-QuIC in \\
CSF or other tissues
\end{tabular}

DefinitesCJD Progressive neurological syndrome and Neuropathologically, or immunocytochemically, or biochemically confirmed

impairment, myoclonus, visual disturbances, cerebellar ataxia, pyramidal and extrapyramidal signs [5]. These symptoms are non-specific and can be caused by a variety of diseases. The patient in this case report, at admission presented blurred vision, walking abnormalities and speech disorders, and her condition progressively deteriorated.

The unspecific clinical presentation of CJD created a need for finding an accurate biomarker of the disease, which proved to be protein 14-3-3. These proteins are ubiquitously expressed in various types of tissues, but their highest expression is in the brain [9]. An elevated level of 14-3-3 protein is most commonly associated with CJD. According to Taim Muayqil, et al., assays for CSF 14-3-3 have sensitivity $\sim 92 \%$ and specificity $\sim 80 \%$ in diagnosing CJD [10]. The presence of 14-3-3 proteins in the CSF may also occur in many other diseases that cause acute or sub-acute neuronal damage, including Alzheimer's disease, stroke, cerebral vasculitis, infectious and paraneoplastic encephalitis, anoxic and metabolic encephalopathy [11-12]. It seemed reasonable that the negative result of 14-3-3 protein reduced the suspicion of $\mathrm{SCJD}$, as happened in the current case. After the negative result of protein 14-3-3 in the CSF, the diagnostic process in the presented patient was misdirected to false trails.

An electroencephalography (EEG) can provide supportive but not definitive evidence for CJD. Typical EEG findings in sCJD consist of periodic sharp wave complexes (PSWC), often referred as the hallmark finding [13-14]. Triphasic waves resembling PSWC, however, may also frequently occur in other neurological or systemic diseases, including metabolic (mostly hepatic) encephalopathy [15]. It is estimated that objective EEG criteria for SCJD have a sensitivity of $64 \%$ and specificity of $91 \%$ [16]. In the presented case, with the progression of the disease, the patient developed a characteristic for CJD EEG containing triphasic sharp waves, delta and theta waves.

MRI has become increasingly important for the clinical diagnosis of sCJD. The most useful in diagnosis of CJD are FLAIR and diffusion weighted imaging (DWI) sequences [17]. The most common MRI change in SCJD is hyperintensity of cortical and basal ganglia. On DWI sequences, hyperintensity 
in the insula and cingulate can often be observed, in addition to the involvement of the cortical grey matter (so-called 'cortical ribboning') near the midline and the superior frontal gyrus [18]. A study by Young et al. showed that DWI and FLAIR imaging were $91 \%$ sensitive, $95 \%$ specific, for diagnosing sCJD [17]. The patient in the current case had normal head MRI up to the third examination (after 3 weeks of hospitalization). It showed a hyperintense signal of thalamus, increased signal intensity in the bilateral caudate nucleus, putamen and gray matter of parietal, temporal and occipital lobes. These changes fulfilled the criteria of sCJD.

It was planned to make the real-time quaking-induced conversion (RT-QuIC) test from the CSF of the patient. Unfortunately, due to the rapid deterioration of her condition, this idea had to be abandoned. RT-QuIC assays are new methods based on prion-seeded amyloid fibril formation by recombinant prion protein (rPrPSen) in multiwell plates [19]. According to the National CJD Research \& Surveillance Unit in Edinburgh, Scotland, UK, this examination has $92 \%$ sensitivity and $100 \%$ specificity [20].

\section{CONCLUSION}

There is considerable variability in the clinical presentation of CJD. The disease is extremely rare which makes the diagnosis of CJD very difficult. The purpose of this case report was to mark the concept that sporadic CJD may mimic stroke at the onset. To further complicate the issue, the 14-3-3 protein can be normal during the disease process. Even a patient with a history of vascular disease, when reporting a rapidly progressive deterioration of cognitive functions, requires differential diagnosis, including CJD.

\section{REFERENCES}

1. Ronnie H, Frederick A. Murphy. Etymologia: Creutzfeldt-Jakob Disease. Emerg Infect Dis. 2017; 23(6): 956. http://doi.org/10.3201/eid2306. ET2306

2. Iwasaki Y. Creutzfeldt-Jakob disease. Neuropathology. 2017; 37(2): 174-188. https://doi.org/10.1111/neup.12355

3. Geschwind MD. Prion Diseases. Neuroinfectious Disease. 2015; 21: 1612-1638. https://doi.org/10.1212\%2FCON.0000000000000251

4. Puoti G, Bizzi A, Forloni G, Safar JG, Tagliavini F, Gambetti P.Sporadic human prion diseases: molecular insights and diagnosis. Lancet Neurol. 2012; 11(7): 618-628. https://doi.org/10.1016/S1474-4422(12)70063-7
5. Zerr I, Poser S. Clinical diagnosis and differential diagnosis of CJD and vCJD. With special emphasis on laboratory tests. APMIS. 2002; 110(1): 88-98. http://doi.org/10.1034/j.1600-0463.2002.100111.x

6. Roos R, Gajdusek DC, Gibbs CJ Jr. The clinical characteristics of transmissible Creutzfeldt-Jakob disease. Brain. 1973; 96(1): 1-20.

7. Geiger KD, Brecht U, Schober R, Schlote W. Creutzfeldt-Jakob-Krankheit: Fehlende Korrelation zwischen zerebraler kortikaler Histologie und klinischem Verlauf der Erkrankung in 22 Obduktionsfällen [CreutzfeldtJakob disease. Lack or correlation between cerebral cortex histology and clinical course of the disease in 22 autopsy cases]. Pathologe. 2002; 23 : 252-259. http://doi.org/10.1007/s00292-002-0541-5

8. National Creutzfeldt-Jakob Disease Surveillance Diagnostic Criteria. National Creutzfeldt-Jakob Disease Surveillance 2017. https://www.cjd. ed.ac.uk/sites/default/files/criteria.pdf (access: 2019.04.04)

9. Martin H, Rostas J, Patel Y, Aitken A. Subcellular localisation of 14-3-3 isoforms in rat brain using specific antibodies. J Neurochem. 1994;63(6): 2259-2265. https://doi.org/10.1046/j.1471-4159.1994.63062259.x

10. Muayqil T, Gronseth G, Camicioli R. Evidence-based guideline: diagnostic accuracy of CSF 14-3-3 protein in sporadic CreutzfeldtJakob disease: report of the guideline development subcommittee of the American Academy of Neurology. Neurology. 2012; 79(14): 1499-1506. https://doi.org/10.1212/WNL.0b013e31826d5fc3

11. Burkhard PR, Sanchez JC, Landis T, Hochstrasser DF.CSF detection of the 14-3-3 protein in unselected patients with dementia. Neurology. 2001; 56(11): 1528-1533. https://doi.org/10.1212/WNL.56.11.1528

12. Huang N, Marie SK, Livramento JA, Chammas R, Nitrini R. 14-33 protein in the CSF of patients with rapidly progressive dementia, Neurology. 2003; 61(3): 354-357. https://doi.org/10.1212/01. WNL.0000078890.89473.ED

13. Furlan AJ, Henry CE, Sweeney PJ, Mitsumoto H. Focal EEG abnormalities in Heidenhain's variant of Jakob-Creutzfeldt disease. Arch Neurol. 1981; 38(5): 312-314. http://doi.org/10.1001/archneur.1981.00510050078015

14. Steinhoff BJ, Racker S, Herrendorf G, Poser S, Grosche S, Zerr I, et al. Accuracy and reliability of periodic sharp wave complexes (PSWC) in Creutzfeldt-Jakob disease. Arch Neurol. 1996; 53(2): 162-166. http:// doi.org/10.1001/archneur.1996.00550020074017

15. Sutter R, Stevens RD, Kaplan PW. Significance of triphasic waves in patients with acute encephalopathy: a nine-year cohort study. Clin Neurophysiol. 2013; 124(10): 1952-1958. https://doi.org/10.1016/j. clinph.2013.03.031

16. Steinhoff BJ, Zerr I, Glatting M, Schulz-Schaeffer W, Poser S, Kretzschmar HA. Diagnostic value of periodic complexes in Creutzfeldt-Jakob disease. Ann Neurol. 2004; 56(5): 702-708. https://doi.org/10.1016/j. clinph.2013.03.031

17. Young GS, Geschwind MD, Fischbein NJ, Martindale JL, Henry RG, Liu S, et al. Diffusion-weighted and fluid-attenuated inversion recovery imaging in Creutzfeldt-Jakob disease: high sensitivity and specificity for diagnosis. AJNR Am J Neuroradiol. 2005; 26(6): 1551-1562.

18. Fragoso DC, Gonçalves Filho AL, Pacheco FT, Barros BR, Aguiar Littig I, Nunes RH, et al. Imaging of Creutzfeldt-Jakob Disease: Imaging Patterns and Their Differential Diagnosis. RadioGraphics. 2017; 37(1): 234-257. https://doi.org/10.1148/rg.2017160075

19. McGuire LI, Peden AH, Orrú CD, Wilham JM, Appleford NE, Mallinson G, et al. Real Time Quaking-Induced Conversion Analysis of Cerebrospinal Fluid in Sporadic Creutzfeldt-Jakob Disease. Ann Neurol. 2012; 72(2): 278-285. https://doi.org/10.1002/ana.23589

20. Green AJE. RT-QuIC: a new test for sporadic CJD. Pract Neurol. 2019; 19(1): 49-55. https://doi.org/10.1136/practneurol-2018-001935 